${ }^{1}$ Centro Universitário de Brusque (Unifebe) Brusque (SC), Brasil. patriciazogbi@hotmail.com

2 Universidade do Vale do Itajaí (Univali) - Itajaí (SC) Brasil.

\section{Educação permanente sobre a atenção psicossocial em situação de desastres para Agentes Comunitários de Saúde: um relato de experiência}

\author{
Permanent education on psychosocial care in disaster situations for \\ Community Health Agents: an experience report
}

Patricia Zogbi dos Santos $\mathbf{1}$, Jones Ivan Dias', Roberta Borghetti Alves²

DOI: 10.1590/0103-11042019S315

RESUMO Tendo em vista a ocorrência de desastres socioambientais e a necessidade de uma atenção psicossocial realizada no território onde residem as pessoas afetadas, elaborou-se uma educação permanente. O objetivo deste relato de experiência foi analisar a realização da educação permanente sobre a atenção psicossocial em situações de desastres para Agentes Comunitários de Saúde(ACS) em um município localizado na Região de Saúde do Médio Vale do Itajaí/Santa Catarina/Brasil. Para realização dessa atividade, utilizaram-se metodologias ativas de modo a abordar temáticas voltadas ao desastre, risco, vulnerabilidade e atenção psicossocial. Como principais resultados, destacam-se a identificação de áreas suscetíveis a ocorrência de desastres localizadas nas microáreas de cada agente, a reflexão sobre a contribuição dos ACS para a realização de uma atenção psicossocial, assim como estabelecimento de ações intersetoriais entre esses profissionais e os agentes da Defesa Civil. Por fim, salienta-se que os recursos utilizados por meio da educação ativa possibilitaram a reflexão do processo de trabalho dos ACS. Sugere-se que nas próximas ações busque-se contemplar toda a equipe de saúde por meio da utilização das reuniões de equipe, como espaço potente para esta ação.

PALAVRAS-CHAVE Atenção Primária à Saúde. Educação continuada. Agentes Comunitários de Saúde. Desastres naturais. Defesa Civil.

ABSTRACT In view of the occurrence of socio-environmental disasters and the need for psychosocial care carried out in the territory where the affected people reside, a permanent education was developed. The objective of this experience report was to analyze the accomplishment of permanent education on psychosocial care in disaster situations for Community Health Agents (CHA)in a municipality located in the Middle Valley Health Region of Itajai/Santa Catarina/ Brazil. To perform this activity, active methodologies were used in order to address issues related to disaster, risk, vulnerability, and psychosocial care. The main results highlight the identification of areas susceptible to the occurrence of disasters located within the micro areas of each agent, the reflection on the contribution of CHA to the accomplishment of psychosocial care, as well as the establishment of intersectoral actions between these professionals and Civil 
Defense agents. Finally, it is emphasized that the resources used through active education made it possible to reflect on the work process of CHA. For the next actions, it is suggested that the entire health team be contemplated through the use of team meetings as a potent space for such action.

KEYWORDS Primary Health Care. Education, continuing. Community Health Workers. Natural disasters. Civil Defense.

\section{Introdução}

Devido às constantes mudanças na sociedade contemporânea em prol do progresso, tem ocorrido a destruição dos recursos naturais. Essa questão torna-se paradoxal na medida em que a dicotomia progresso $\mathrm{x}$ incidência de desastres tem acarretado riscos para a população que se vê afetada devido às perdas de familiares, amigos, de moradias e bens materiais, bem como a existência de poucas de políticas públicas voltadas a essas pessoas'.

Diante disso, faz-se necessário compreender o que são desastres e quais as suas implicações para a vida dos indivíduos. Para melhor compreensão do desastre, deve-se elucidar dois conceitos importantes, o risco e a vulnerabilidade, que atuam mutuamente na incidência dele. O risco caracteriza-se pela probabilidade ou pela iminência de um desastre; já a vulnerabilidade caracteriza-se pelas condições de uma comunidade, provenientes de fatores socioeconômicos, ambiental, cultural, físico, que a tornam propensa à incidência de desastres ${ }^{2}$. Assim, o desastre é a relação bilateral de risco e vulnerabilidade, isto é, trata-se de um evento que extrapassa o limite do risco, efetivando-se em um ambiente vulnerável, de modo a alterar o funcionamento e o modo de levar a vida da comunidade afetada ${ }^{3}$.

Seguindo essa premissa, faz-se necessário abordar o papel da atenção psicossocial em situações de desastres, de modo a integrar ações que busquem a reintegração do sujeito como ser biopsicossocial e que promovam a coesão social ${ }^{4}$, a fim de compreender que, nem todas as pessoas afetadas por tal evento estarão traumatizadas, porém, cada indivíduo necessitará de um cuidado singular visando a um menor sofrimento psíquico. Como o Sistema Único de Saúde (SUS) é o serviço que realiza tal ação, é necessário destacar o papel da Atenção Básica (AB) nesse processo. Assim, antes da ocorrência de desastres, é necessário que a equipe da $\mathrm{AB}$ esteja integrada com a perspectiva psicossocial, visto que ser afetado pelo desastre ocasiona a perda de referências socioafetivas, de bens, de referências geográficas e de sensação de segurança. Além disso, depara-se com a imprevisibilidade, pode-se sentir medos intensos e sensação de impotência diante do desastre, bem como vivenciar uma mudança de tarefa e de papéis dentro da família 5 .

Assim, compreende-se que os primeiros cuidados psicossociais na ocorrência de desastres são essenciais, visto que eles tencionam reduzir o estado de estresse, com o objetivo de prevenção de patologias. Também auxiliam sujeitos que apresentam dificuldade em elaborar e ressignificar a experiência do evento; contribuem para o enfrentamento das dificuldades atreladas à mudança de rotina; ajudam no alojamento temporário e as famílias a encontrarem momentos privativos ${ }^{5}$. Dessa forma, torna-se necessário que as formações voltadas à atenção psicossocial ocorram com frequência, visando a uma atuação mais eficaz na ocorrência de um desastre ${ }^{5}$. Para 
tais formações, devem ser abordados temas específicos da atenção psicossocial, contendo: estratégia de intervenção psicossocial na ocorrência de desastres; primeiro acolhimento psicossocial; o cuidar de si mesmo; formas de ajudar um indivíduo em sofrimento e sintomas psicológicos pós-desastre ${ }^{5}$.

A partir dessas possibilidades de intervenção voltada à atenção psicossocial, destaca-se a necessidade de, se possível, realizar esse suporte no local onde a comunidade reside a fim de que, aos poucos, seja ressignificado o ocorrido. Nesse sentido, destaca-se a $\mathrm{AB}$ por meio da Estratégia Saúde da Família (ESF) e do Núcleo Ampliado de Saúde da Família e Atenção Básica (Nasf-AB) como um ponto de atenção importante para realização desse suporte.

Conhecida como a porta de entrada dos usuários do SUS, a $\mathrm{AB}$ caracteriza-se pelo conjunto de ações de saúde individuais, familiares e coletivas que envolvem a promoção, a prevenção, a proteção, o diagnóstico, o tratamento, a reabilitação, a redução de danos, os cuidados paliativos e a vigilância em saúde, desenvolvida por meio de práticas de cuidado integrado ${ }^{6}$. No que concerne à ESF, é importante destacar que os profissionais possuem algumas atribuições comuns e específicas. Nessa lógica, deve fazer parte do processo de trabalho de cada profissional que compõe essa equipe fazer um diagnóstico do território de atuação; realizar o acolhimento com escuta apropriada; realizar atendimentos domiciliares; promover a atenção integral e desenvolver ações educativas que possam interferir no processo de saúde-doença da população; identificar parceiro e recursos na comunidade que possam potencializar as ações intersetoriais, tais como igrejas, escolas e associações ${ }^{7}$.

Dentre os profissionais que estão inseridos na ESF, destaca-se o Agente Comunitário de Saúde (ACS), que trabalha na microárea onde a população reside, de modo a realizar cadastramento domiciliar, atividades de promoção da saúde e prevenção das doenças e dos agravos, além de práticas de vigilância à saúde por meio de atendimentos domiciliares e ações educativas individuais e coletivas nos domicílios e na comunidade ${ }^{8}$. Assim, a AB possui um papel importante na gestão de risco, coordenando o cuidado à população afetada, já que o conhecimento do território e vínculo com a população auxilia também a atuação dos profissionais de emergência, ou seja, os ACS podem vir a ser o elo entre a comunidade e os demais setores, como, por exemplo, a Defesa Civil4

Desse modo, ressalta-se a importância do preparo dos profissionais da $\mathrm{AB}$ para realizar atenção psicossocial nas situações de desastres. Para tal formação, não basta apenas desenvolver técnicas e procedimentos, isto é, deve-se investir em formação para que a aplicação do que fora aprendido seja eficaz, pensando assim em uma articulação e uma colaboração mútua entre instituições, técnicos e população afetada ${ }^{4}$. Destaca-se a educação permanente como uma ferramenta de tecnologia leve que se caracteriza por um processo educativo direcionado ao trabalho, sendo aplicado no cotidiano dos profissionais de saúde. Essa educação pauta-se a partir das problemáticas experienciadas, levando em conta as vivências e o conhecimento que os colaboradores já possuem. Assim, a educação permanente tem por objetivo qualificar profissionais de saúde, evidenciando as necessidades de saúde da população e do controle social. As práticas, portanto, devem ser direcionadas à atenção integral, a fim de preencher lacunas e transformar as práticas profissionais para a melhoria de qualidade de vida dos usuários e da comunidade?

Tendo em mente a importância das ações multiprofissionais em contextos de desastres, bem como a elaboração de iniciativas de Educação Permanente em Saúde que levam em conta a necessidade locorregional e que propiciem práticas colaborativas intersetoriais e interprofissionais que promovam a saúde da população, faz-se necessário abordar a educação permanente, processo do qual possibilitará a articulação dos profissionais da $\mathrm{AB}$ e da Defesa Civil para a gestão de risco e de desastres. Desse modo, o objetivo deste relato de experiência é analisar criticamente 
a educação permanente realizada com ACS em um município localizado na Região de Saúde do Médio Vale do Itajaí/Santa Catarina/Brasil sobre atenção psicossocial em situações de desastres.

\section{Material e métodos}

Este trabalho foi desenvolvido em um estágio específico do nono período de um Curso de Psicologia em uma faculdade localizada no Vale do Itajaí/Santa Catarina. Os acadêmicos realizaram o estágio no Nasf e, a partir das demandas identificadas no território de uma ESF, elaboraram um projeto de educação permanente voltado à temática atenção psicossocial em situações de desastres. Para elaboração e condução desse projeto, contou-se com o apoio da coordenação da Defesa Civil do município a fim de que pudesse realizar uma ação intersetorial de modo a articular a Política Nacional de Atenção Básica e a Política Nacional de Proteção e Defesa Civil.

Para elaborar as metodologias dos encontros, baseou-se na educação ativa, na qual utiliza-se de instrumentos que possibilitem a reflexão e a criação de estratégias para melhora do processo de trabalho. A educação ativa é uma metodologia que permite intercalar a teoria com a prática das temáticas apresentadas e favorece a autonomia dos participantes, despertando a curiosidade deles e estabelecendo com que os constituintes se posicionem de forma crítica ${ }^{10}$. No que concerne à importância da educação ativa, Bueno ${ }^{11}$ ressalta que, a partir dos conhecimentos prévios dos participantes, é possível levá-los a uma melhor compreensão sobre o tema abordado, visto que os participantes estarão em contato com suas sensações e percepções, facilitando, assim, o processo de ensino-aprendizagem.

A educação permanente ocorreu em uma Unidade Básica de Saúde localizada em um município da Região de Saúde do Médio Vale do Itajaí. Foi conduzida por dois acadêmicos de Psicologia sob norteamento da professora orientadora em conjunto com o coordenador e agentes da Defesa Civil do município. Teve-se a participação de oito agentes comunitários de saúde, sendo, destes, seis mulheres e dois homens com faixa etária entre 30 e 50 anos. Foram realizados nove encontros entre os meses de abril e junho de 2017, os quais ocorreram uma vez por semana, tendo duração de duas horas por encontro.

A fim de compreender o que os ACS já possuíam de conhecimento sobre o tema proposto, bem como de verificar o conhecimento adquirido ao término da educação permanente, aplicou-se um roteiro de entrevista semiestruturada no primeiro e no último encontro. $\mathrm{O}$ roteiro consistiu em nove perguntas, tais como: 1) O que é um desastre para você?; 2) O que é para você Defesa Civil?; 3) Quais são as ações que a Defesa Civil realiza?; 4) Para você, o que é uma área suscetível à ocorrência de desastres?; 5) Para você, qual seria o papel da AB nas etapas de Prevenção, Mitigação e Preparação de desastre?; 6) Quais as intervenções psicossociais realizadas pela equipe de $A B$ nos pré-desastres?; 7) Na sua opinião, qual o papel da $A B$ no manejo (resposta) de desastres?; 8) Quais as primeiras intervenções psicossociais realizadas pela equipe de $\mathrm{AB}$ nos pós-desastres?; 9) Quais as primeiras intervenções psicossociais realizadas pela equipe de $\mathrm{AB}$ na recuperação pós-desastres?

A educação permanente ocorreu por meio da construção de temáticas condizentes com a realidade dos participantes. Nas temáticas voltadas aos desastres, foram realizados quatro encontros. Neles, foram dialogados sobre as ações realizadas pela Defesa Civil, em que o coordenador do respectivo órgão explanou sobre as fases de atuação para uma gestão integral do risco e do desastre. Posteriormente, foi abordado o que é o desastre, o risco e a vulnerabilidade por meio da construção de uma maquete. Os acadêmicos orientaram para que os ACS montassem uma maquete sobre o que julgavam por área de risco de inundação e deslizamento, a fim de considerarem os conhecimentos adquiridos pelos participantes. No terceiro encontro, com a finalidade de 
identificar áreas de risco e vulnerabilidade, realizou-se, por meio de um mapeamento do território, a identificação das áreas de risco que os ACS visitam. Tal identificação fora realizada com o auxílio da Defesa Civil. No quarto encontro, realizaram-se visitas às principais áreas suscetíveis à ocorrência de desastre que são atendidas pelos ACS, com o intuito de explicar os critérios utilizados pela Defesa Civil para identificar e classificar uma área de risco e como ocorre a interdição do local.

$\mathrm{O}$ quinto e sexto encontro voltaram-se às temáticas direcionadas às ações da ESF. No quinto encontro, explicitaram-se as intervenções que devem ser realizadas na fase de pré-desastre, por meio de recursos audiovisuais, bem como roda de conversa. No sexto, os acadêmicos mensuraram a importância do papel da $\mathrm{AB}$ no manejo do desastre, bem como quais atividades os ACS poderiam realizar nessa fase.

No tocante à atenção psicossocial, realizaram-se dois encontros. No sétimo encontro, realizou-se uma discussão acerca dos Primeiros Cuidados Psicológicos (PCP), em que buscou-se sensibilizar os participantes sobre a relevância em ofertar um apoio psicossocial às pessoas em sofrimento psíquico por conta da ocorrência de desastres. Na discussão, foram transmitidos conceitos, objetivos bem como benefícios do PCP, por meio de recursos audiovisuais, role-play e roda de conversa. No oitavo encontro, visou-se discutir com os ACS a respeito das intervenções psicossociais nos pós-desastres. Ademais, realizou-se a aplicação do mesmo roteiro de entrevista semiestruturada aplicado no primeiro encontro a fim de verificar se houve o aumento do conhecimento sobre as temáticas abordadas.

\section{Resultados e discussões}

Tendo em vista os aspectos observados, destaca-se que foi possível, a partir das vivências profissionais dos ACS quanto à atenção psicossocial, sensibilizá-los para a relevância da participação e integração das políticas públicas voltadas à $\mathrm{AB}$ e à Defesa Civil, promover a reflexão dos ACS com relação à situação de desastres, construir propostas de intervenções intersetoriais em situações de desastres e estimular a participação dos ACS em ações coletivas com a Defesa Civil.

No que concerne à análise dos resultados da primeira entrevista realizada, identificou-se que os oito participantes descreveram um desastre como situações inesperadas, sendo essas causadas por desmatamentos, enchentes e deslizamentos. consistem relação à Defesa Civil, destacaram que tal instituição atua apenas no momento da ocorrência do desastre, ou seja, participando de uma perspectiva mais reducionista da gestão de riscos e de desastres. Nota-se que a questão que obteve respostas similares fora a número quatro, em que se buscava compreender o nível de conhecimento sobre áreas de risco e vulnerabilidade. Compreende-se que tal questão obteve respostas similares devido aos conceitos de risco e vulnerabilidade já estarem presentes no cotidiano dos ACS. Nesse sentido, Noal et al. ${ }^{5}$ relatam que as ações cotidianas dos ACS possibilitam que eles identifiquem, a partir das visitas domiciliares e do acompanhamento das microáreas de risco, os riscos e vulnerabilidades de cada local.

Já a análise da questão cinco demonstra que os ACS estão cientes de seu papel com relação à prevenção, mitigação e preparação de desastres. Observou-se que eles responderam de forma similar à questão, descrevendo que o papel da $\mathrm{AB}$ é de orientar os usuários a atentarem para os alertas realizados por meio da rádio local, bem como alertar a população sobre os perigos e as consequências das enchentes e dos deslizamentos. Além disso, os ACS consideram essencial realizar a prevenção ao longo do ano, que pode ser feita em encontros com a população das áreas de risco. Rocha ${ }^{12}$ elucida que as ações cotidianas dos ACS devem contemplar o diálogo com os usuários que residem em áreas de risco, mapear grupos vulneráveis que teriam mais dificuldade de deslocamento em uma situação de desastre, bem como uma comunicação ativa entre ACS e Defesa Civil. 
No que concerne às questões seis e sete, deve-se destacar que essas foram respondidas por um ACS. A questão seis buscava compreender quais os conhecimentos prévios dos ACS com relação às intervenções psicossociais realizadas pela equipe de $\mathrm{AB}$ no pré-desastre. Para essa questão, a resposta fora o cuidado e o acompanhamento das vítimas. Já na questão sete, relacionada com o papel da $\mathrm{AB}$ no manejo de desastres, os ACS responderam ser necessário atentar para as vítimas e direcioná-las a um local seguro. É pertinente frisar que as questões oito e nove ficaram sem resposta, o que demonstra a importância em abordar a atenção psicossocial.

Abaixo, encontram-se os resultados por principais temáticas que foram abordadas na educação permanente.

\section{Desastres}

No que diz respeito aos resultados dessa temática, após a construção da maquete, os acadêmicos questionaram os ACS sobre os motivos de colocarem casas ao lado do rio e do talude. Assim, os participantes deixaram claro que identificaram como áreas de risco casas próximas ao rio em virtude de enchentes. Já com relação ao talude, mencionaram sobre os riscos de deslizamento. Além disso, duas ACS mencionaram o fato de muitos moradores perderem todos seus bens materiais: "Essa situação é muito triste, já vi pessoas perderem tudo o que tinham por morarem em áreas de risco". Com relação à favela inserida na maquete, os ACS elucidaram que elas são consideradas áreas de risco devido à infraestrutura precária desses locais.

Observou-se que os ACS compreenderam o tema apresentado, pois todos participaram da realização da maquete, especificando os locais considerados de risco e vulneráveis à ocorrência de desastres, bem como discorrendo sobre quais desastres poderiam ocorrer nessas áreas consideradas de risco.

Em relação à identificação de áreas de risco e vulnerabilidade, pode-se afirmar que a participação dos ACS foi ativa, realizando diversas perguntas ao coordenador da Defesa Civil, como, por exemplo, sobre quais as cotas de água de cada rua que é necessário obter para ocorrer um alagamento, a fim de informar aos seus usuários posteriormente. Dessa forma, os ACS atentaram em compreender o porquê tais áreas são consideradas de risco. Entre as áreas verificadas, identificaram-se duas áreas de risco dentro de seus territórios, visto que ambas possuem risco de enchente.

O coordenador da Defesa Civil explicou os critérios utilizados por esse órgão para identificar e classificar uma área de risco e como ocorre a interdição do local. Observou-se que os ACS obtiveram conhecimento da explicação do coordenador, pois, ao serem questionados, no final do encontro, sobre o porquê de a área visitada ser considerada uma área de risco, eles conseguiram elucidar de forma correta o motivo durante a visita pelo local. Assim, os ACS identificaram que o local visitado possuía evidências de movimentação, desmatamento e inclinação de terreno.

\section{Estratégia Saúde da Família}

Observou-se que as informações anunciadas acerca da gestão de desastres foram compreendidas, visto que, ao serem questionados com relação à gestão de desastres, os ACS relataram que "É necessário realizar drenagem”. Outro ACS salientou que "é nosso papel alertar os usuários, nós somos os olhos da AB”. Por fim, um participante ressaltou

claro que não basta só avisar, nós devemos estar em alerta constante, verificando os meios de comunicação, alertando a população sobre as cotas de água e repassar os meios de comunicação que a Defesa Civil utiliza para alertar a população.

Notou-se que os ACS adquiriram conhecimento sobre a temática apresentada, visto que contribuíram para ela com estratégias de prevenção e manejo de desastres nas situações apresentadas. 
Além disso, constatou-se que os ACS compreenderam a relevância do tema, uma vez que comentaram sobre muitos usuários que vivem em situações de vulnerabilidade social, porém, acreditam que, talvez por ser o único lugar que os indivíduos têm como moradia, eles acabam convivendo com o risco. A partir disso, fora frisada a importância do mapeamento de risco para que os ACS se tornem agentes multiplicadores de conhecimento.

\section{Atenção psicossocial}

Realizou-se uma discussão acerca dos PCP visando sensibilizar os participantes sobre a relevância de oferecer um apoio às pessoas em sofrimento psíquico por conta da ocorrência de desastres. Durante a discussão, um ACS fez o seguinte comentário

Se eu prestar apoio as vítimas, vou perguntar se ela precisa de algum auxílio, ofereço minha ajuda, vou perguntar se ela quer dizer o que está sentindo e claro, vou respeitar se ela não quiser falar.

Com essa manifestação do participante e tendo em vista como é realizado o PCP, nota-se que os Agentes adquiriram conhecimento sobre a temática. Após a discussão, realizou-se uma simulação de PCP, de forma que os ACS conseguiram acolher de forma empática o sujeito afetado pela enchente.

Seguindo essa premissa, para que fosse possível obter conhecimento sobre a atenção psicossocial, foi necessário abranger todas as fases da Defesa Civil, construindo estratégias de prevenção, mitigação, preparação e resposta aos desastres, pois a atenção psicossocial engloba o entendimento das fases de atuação na gestão de risco. Notou-se que os participantes obtiveram conhecimento da temática por meio do roteiro de entrevista semiestruturada no qual os ACS aumentaram o nível de conhecimento no decorrer dos encontros, conforme observado na análise dos dados.

No que concerne às temáticas abordadas e às metodologias utilizadas, observou-se que tais práticas possibilitaram a interação e a construção do conhecimento entre os participantes e os preponentes do projeto. Assim, pode-se relacionar as respostas dos ACS com a entrevista realizada por Rocha ${ }^{12}$ em sua tese, em que um profissional da saúde relata que os ACS desejam participar de ações em conjunto com a Defesa Civil, pois estão envolvidos diretamente com a comunidade e com as áreas mais vulneráveis, assim, as equipes de saúde se sentem motivadas em atuar em parceria com esse órgão. Tal depoimento sinaliza o envolvimento e a seriedade do trabalho desenvolvido no cotidiano do trabalho do ACS.

\section{Considerações finais}

Por meio da educação permanente, foi possível analisar a educação permanente realizada com ACS sobre atenção psicossocial em situações de desastres. Identificou-se que os recursos utilizados por meio da educação ativa possibilitaram a reflexão do processo de trabalho dos ACS. Além disso, houve o aumento do conhecimento como pode ser identificado por meio dos resultados advindos do roteiro de entrevista semiestruturada.

Por intermédio da educação permanente, identificaram-se, em conjunto com a Defesa Civil, as principais áreas suscetíveis de ocorrência de desastres que são cobertas pela ESF e que recebem o atendimento domiciliar do ACS. Assim, pode-se estabelecer parcerias por meio do acordo de ações intersetoriais entre os ACS e os agentes da Defesa Civil. Acordou-se que, caso os agentes percebam que há alguma alteração das características física da moradia ou do terreno, de modo a contribuir para suscetibilidade da ocorrência do desastre, estes irão dialogar com a população e buscarão auxílio da Defesa Civil para realizar estratégias de mitigação para redução de riscos de desastres.

No tocante à atenção psicossocial, desmitificou-se o tabu que esta ação só poderia ser realizada pela psicologia, de modo a destacar a importância da equipe de saúde para esta, 
bem como para a reflexão sobre a contribuição dos ACS para a realização de uma atenção psicossocial, assim como estabelecimento de ações intersetoriais entre estes profissionais e os agentes da Defesa Civil.

Destaca-se a importância da utilização de instrumentos que possibilitem verificar a eficácia da educação permanente de modo a ser aplicado no primeiro e no último encontro. Sugere-se que sejam utilizados instrumentos de coleta de dados nas educações permanentes como forma de avalição do método educativo.

Como fragilidade dessa educação permanente, destaca-se a realização dessa ação voltada somente aos ACS. Sugere-se que nas próximas ações busque-se contemplar toda a equipe de saúde por meio da utilização das reuniões de equipe, como espaço potente para essa ação. Por fim, diante da nova Política Nacional de Atenção Básica que traz insegurança para os ACS, reitera-se a importância das ações desenvolvidas por esse profissional, pois ele é o elo entre a comunidade e AB, bem como é um profissional fundamental para mapear os líderes comunitários, realizar ações intersetoriais com a Defesa Civil e, assim, contribuir para a gestão integral de riscos e desastres.

\section{Colaboradores}

Santos PZ (0000-0002-6864-7770)*, Dias JI (0000-0002-1101-303X)* e Alves RB (00000002-1866-699X)* contribuíram igualmente na realização das seguintes atividades: 1) concepção e o planejamento do manuscrito, assim como análise e a interpretação dos dados; 2) elaboração do manuscrito e revisão crítica do conteúdo; e 3) aprovação da versão final do manuscrito.

\section{Referências}

1. Alves RB, Lacerda MAC, Legal EJ. A atuação do psicólogo diante dos desastres naturais: uma revisão. Psicol. Estud. [internet]. 2012 [acesso em 2017 set 4]; 17(2):307-315. Disponível em: http://www.scielo. br/pdf/pe/v17n2/v17n2al3.pdf.

2. Coelho AEL. Percepção de Risco no Contexto da Seca: Um Estudo Exploratório. Psicol. Am. Lat. [internet]. 2007 [acesso em 2017 abr 15]; 1(10):1-6. Disponível em: http://pepsic.bvsalud.org/scielo.php?script=sci_ arttext\&pid=S1870-350X2007000200012\&lng=pt\&t $\operatorname{lng}=\mathrm{pt}$.
3. Favero E, Sarriera JC, Trindade MC. O Desastre na Perspectiva Sociológica e Psicológica. Psicol. Estud. [internet]. 2014 [acesso em 2017 abr 15]; 19(2):201209. Disponível em: http://www.scielo.br/pdf/pe/ v19n2/04.pdf.

4. Benevides LRS. A Atenção Psicossocial e as intervenções geradas em contextos de desastre: a experiência de profissionais em Teresópolis [dissertação] [internet]. Rio de Janeiro: Escola Nacional de Saúde Pública Sergio Arouca, Fundação Oswaldo Cruz; 2015. 76 p. [acesso em 2017 maio 22]. Disponível em: https:// www.arca.fiocruz.br/handle/icict/13792.
${ }^{\star}$ Orcid (Open Researcher and Contributor ID). 
5. Noal DS, Oliveira SS, Alpino TMA, et al. Gestão local de desastres naturais para a atenção básica. São Paulo: Una SUS; 2016.

6. Brasil. Portaria $n^{\circ} 2.436$ de 21 de setembro de 2017 . Aprova a Política Nacional de Atenção Básica, estabelecendo a revisão de diretrizes para a organização da Atenção Básica, no âmbito do Sistema Único de Saúde (SUS). Diário Oficial da União. 22 Set 2017.

7. Mendonça CS. Saúde da família, agora mais do que nunca! Ciênc. Saúde Colet. [internet]. 2009. [acesso em 2017 abr 15]; 14(1):1493-1497. Disponível em: http://www.scielo.br/pdf/csc/v14sl/a22v14s1.pdf.

8. Viana ALD, Dal Poz MR. A reforma do sistema de saúde no Brasil e o Programa de Saúde da Família. Physis [internet]. 1998 [acesso em 2017 abr 18]; 8(2):11-48. Disponível em: http://http://www.scielo.br/scielo. php?pid=S0103-73311998000200002\&script $=$ sci abstract\&tlng=pt.

9. Sarreta FO. Educação permanente em saúde para os trabalhadores do SUS. São Paulo: Unesp; 2009.
10. Borges TS, Alencar G. Metodologias ativas na promoção da formação crítica do estudante: o uso das metodologias ativas como recurso didático na formação crítica do estudante do ensino superior. Cairu em Rev. [internet]. 2014 [acesso em 2017 maio 22]; 3(4):119-143

11. Bueno MA. A Importância do Estudo do Meio Na Prática de Ensino em Geografia Física. BGG [internet]. 2009 [acesso em 2017 maio 20]; 29(2):185-198. Disponível em: https://www.revistas.ufg.br/bgg/article/view/9028.

12. Rocha V. O papel do Agente Comunitário de Saúde na prevenção de desastres naturais por deslizamento em comunidades da cidade do Rio de Janeiro [tese]. Rio de Janeiro: Escola Nacional de Saúde Pública Sergio Arouca, Fundação Oswaldo Cruz; 2009. 204 p .

Recebido em 30/04/2019

Aprovado em 05/09/2019

Conflito de interesses: inexistente

Suporte financeiro: não houve 